\title{
Towards leveraging discrete grid flexibility in chance-constrained power system operation planning
}

\author{
Efthymios Karangelos and Louis Wehenkel \\ Department of EE \& CS - Montefiore Institute, University of Liège, Liège, Belgium \\ \{e.karangelos;l.wehenkel\}@uliege.be
}

\begin{abstract}
This paper considers the integration of grid flexibility in the chance-constrained power system operation planning framework. The particular challenge addressed comes from the discrete nature of the respective controls, such as breaker positions defining the topology of the network. We consider a template short-term operation planning problem statement, seeking to enable $\mathrm{N}-1$ secure operation over a distribution of power injections. We use a scenario-based approach to determine a planning decision and rely on theoretical results to compute an upper bound on the probability of being able to meet the $\mathrm{N}-1$ criterion in operation. We also estimate the actual value of this probability through Monte Carlo simulation. Our results indicate that both the bound and the actual value consistently decrease when increasing the size of the considered scenario set, even if the bound is quite conservative. Moreover, we showcase that further from economic efficiency, grid flexibility can lead to gains in operational reliability.
\end{abstract}

Index Terms-chance-constrained planning, grid flexibility, scenario-based methods, optimal power flow under uncertainty.

\section{INTRODUCTION}

In the energy transition era, managing power injection uncertainty is a major research theme on the fundamental optimal power flow (OPF) [1] problem. While progress is made in the field of robust optimization, extreme conservativeness is typically too costly to afford. Chance-constrained optimization, seeking to ensure that the grid shall remain functional with high enough probability, is a more general approach allowing to avoid such economic burden while holding a desirable explicit guarantee of operability.

To date, most notable contributions to the chanceconstrained OPF (CC-OPF) literature invoke linearity and/or convexity modeling assumptions on the power system, as per the properties of respective solution approaches. The works in [2], [3] adopt the linear DC power flow approximation and the convex SDP relaxation respectively to employ the 'scenarioapproach' from [4] while ensuring the probability of achieving N-1 security. Reference [5] exploits the linearity of DC power flow to analytically reformulate individual chance-constraints expressing the system operational limits, while in [6] this approach is tested in the $\mathrm{AC}$ context by linearizing the power flow model around a forecasted operating point (i.e., assuming that the impact of uncertainty on the power flows is linear). Sundar et al. [7] introduced a comprehensive unit commitment framework taking into account injection uncertainty and $\mathrm{N}$ 1 contingencies. The reformulation approach as per [5] is invoked to handle chance-constraints on power flows and reserve adequacy. Finally, reference [8] adopts the non-convex AC-QP physical model while evaluating only a posteriori the constraint violation probability, on the basis of the theoretical results introduced in [9].

Our research work is motivated by the fact that while grid flexibility (e.g., topology changes, phase-shifting transformers, etc.) is indispensable to modern power system operation, discrete power flow controls remain outside the realm of the stateof-the-art of chance-constrained power system operational planning. With this in mind, we focus on a short-term power system operational planning problem under injection uncertainty, subject to $\mathrm{N}-1$ security constraints and while explicitly modeling discrete grid flexibility. Our goal is to develop an efficient chance-constrained framework for tackling power systems planning problems with such features. As a first step, in this paper we seek to interpret in a power systems context the theoretical result from [10], providing an a posteriori upper bound on the constraint violation probability implied by the solution of a scenario-program. More specifically, we explore the potential to attain theoretical probabilistic guarantees of relevant order of magnitude and the practical substance of said guarantees for power system operation planning applications.

To do so, we study a representative set of operation planning scenario-program instances, progressively analyzing the effect of the size of the scenario set under consideration and the availability of grid flexibility on the probability of feasible N-1 secure system operation. For each solution instance, we compute the theoretical constraint violation probability upper bound as in [10] and we estimate the actual constraint violation probability by means of Monte Carlo simulations. We find that, for the problem under consideration, the theoretical upper bound values appear conservative with respect to the actual values. Moreover, we also find that, while reducing economic cost, using grid flexibility implies a greater probability of achieving N-1 secure power system operation.

The remainder of this paper is organized as follows. Section III describes the power system operation planning problem under consideration while the algorithmic framework developed to facilitate our studies is presented in section III. The study results are introduced and discussed in section IV. We conclude the main body of the paper with section $\mathrm{V}$, while also discussing the outline of an iterative algorithmic approach, towards solving chance-constrained operation planning with 
discrete controls on grid flexibility. Finally, the paper appendix includes the mathematical formulation of the scenario-program under consideration.

\section{PROBLEM DESCRIPTION}

We consider the mission of planning to enable the secure operation of the system for a single forthcoming period under uncertainty on the nodal power injections/demands and on the occurence of transmission component outages. More specifically, to achieve secure operation with (high) probability, we sample scenarios of nodal power injections/demands i.i.d. from a given probability model, and the scenario-program aims at identifying minimum cost operational planning decisions, such that for all gotten scenarios the system can be operated while complying with the $\mathrm{N}-1$ security criterion. The resulting problem formulation is the scenario-based counterpart of the robust formulation in [11].

To model operation seeking to comply with the $\mathrm{N}-1$ security criterion, we represent both preventive and corrective control stages. The former stage responds to the realization of the true nodal injections while the network remains intact, and, given such realizations, the latter responds to the occurrence of a transmission element outage. At both these stages, we explicitly model closed-loop controls as in (i.) generators balancing the mismatch between their dispatch and realized net load as prescribed by automatic generation control (AGC) participation factors, and (ii.) phase-shifting transformers (PSTs) injecting phase-angle differences as per rule-based operating principles 1 . In corrective mode, we consider that the operator may adapt the network topology through opening/closing bus splitting breakers, with a possible limit on the admissible number of simultaneous actions.

We define the task of the operational planner to find firststage decisions, i.e. a minimum cost 'base-case' generation dispatch, AGC participation factors, and a 'base-case' network topology, while jointly anticipating (a.) power injection scenarios, and, (b.) network component outages, and, (c.) the action of closed-loop controls, and, (d.) the recourse topological that could be carried out by the operator in post-contingency mode. The set of the problem constraints includes,

- bounds on AGC participation factors;

- for all injection scenarios:

- generation lower \& upper bounds;

- for the intact network and all credible component outages:

* nodal power balance \& power flow ratings;

* disjunctive inequalities expressing the operating modes of PSTs;

* disjunctive inequalities modeling the position of bus splitting breakers;

* connectivity constraints enforcing that no part of the system is islanded;

\footnotetext{
${ }^{1}$ As detailed in $\S$ III.C of $[11]$ and the appendix of this paper.
}

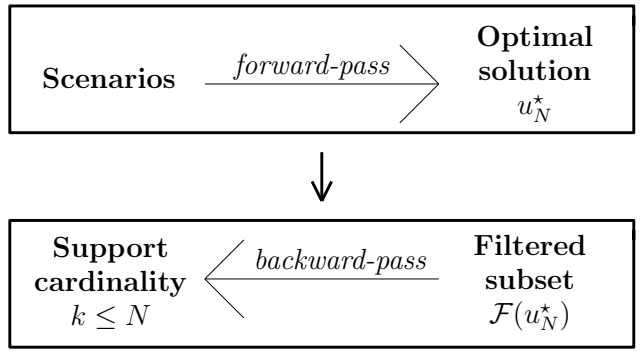

Fig. 1. Algorithmic framework overview

- coupling constraints limiting the number of admissible breaker position changes between the preventive and any corrective stage.

Adopting the linear DC-power flow approximation the aforementioned problem can be cast as a Mixed-Integer Linear Programming (MILP) problem, the detailed statement of which is presented in appendix $B$

\section{ALGORITHMIC FRAMEWORK}

This paper concerns the practical interpretation of the theoretical results from [10] in a power systems operational planning context. The results in [10] provide, under very loose conditions, upper bounds on how well the optimal solution $\left(u_{N}^{\star}\right)$, gotten from of a scenario-program with $N$ scenarios i.i.d. along a certain probability distribution, generalizes to other scenarios drawn according to the same distribution.

To this end, [10] considers the notion of support subset of the scenario-program, i.e., a subset of the full set of $N$ scenarios that would yield the same solution $\left(u_{N}^{\star}\right)$. They show that if we can find a support subset of $k$ scenarios then the probability that $\left(u_{N}^{\star}\right)$ is infeasible for independent scenarios gotten from our generative model is upper bounded ${ }^{2}$ by

$\varepsilon\left(u_{N}^{\star}\right)=\left\{\begin{array}{l}1, \quad \text { if } \quad k=N, \\ 1-\sqrt[N-k]{\frac{\beta}{N\left(\begin{array}{c}N \\ k\end{array}\right)},} \quad \text { else. }\end{array}\right.$

For a given value of $N$, the smaller $k$ the smaller this bound; conversely for a given value of $k$, the larger $N$ the smaller the bound. The smaller $\beta$, the larger the bound; however the influence is not very strong, so that in practice one can use quite small values of $\beta$ (see [10], for further details).

With this in mind, we developed an algorithmic framework that targets the identification of both the optimal solution of a given scenario-program as well as an as small as possible support subset of it.

\section{A. Overview}

The overarching structure of our framework is illustrated in Fig. 1. In a first forward-pass, we start with an empty

\footnotetext{
${ }^{2}$ Here $1-\beta$ is a confidence level, meaning that the upper bound will be satisfied for any given problem for a proportion of at least $(1-\beta)$ of the scenario sets of size $N$ that could be obtained by sampling i.i.d. from the target distribution.
} 
scenario subset and grow its members while updating $1^{\text {st }}$ stage decisions, until no other constraint violating scenario can be identified. Since the objective function only depends on $1^{\text {st }}$-stage decisions, the termination of this step returns the optimal solution to the scenario program under consideration. Moreover, the subset of injection scenarios progressively filtered to identify the said optimal solution is also a support subset to the scenario-program under consideration. However, it remains possible that the latter subset could be reducible, as several of its members may dominate other members. To address this eventuality, in a second backward-pass we attempt to identify and remove dominated scenarios from the filtered subset. Given the final support set cardinality resulting from these two steps, we compute the upper bound on the constraint violation probability as per (1).

\section{B. Forward-pass: growing a support subset}

As a first step, we build the optimal solution of the considered scenario-program as well as a (potentially reducible) first support subset by means of a column \& constraint generation approach [12]. To do so, we initialize the program $1^{\text {st }}$-stage decisions $\left(u_{0}^{\star}\right)$ while only taking into account the forecast injections, and the set of filtered scenarios including only the forecast injections as $\left(\mathcal{F}_{0}\right)$. At each iteration $j \geq 1$, given the latest $1^{\text {st }}$-stage decisions $\left(u_{j-1}^{\star}\right)$ we:

1) assess the feasibility of all unfiltered $N-(j-1)$ injection scenarios, by solving in parallel sub-problems quantifying the respective degree of constraint violation,

2) include the most-infeasible injection scenario $\left(i_{j}\right)$ to a larger subset of filtered scenarios $\mathcal{F}_{j}=\mathcal{F}_{j-1} \cup i_{j}$,

3) update $1^{\text {st }}$-stage decisions $\left(u_{j}^{\star}\right)$ while solving a scenario-program $v s$ the filtered subset $\mathcal{F}_{j}$,

4) return to step 1, until no further constraint-violating injection scenario is identified,

5) define the optimal solution as $u_{N}^{\star}=u_{j}^{\star}$ and the respective filtered scenario subset as $\mathcal{F}\left(u_{N}^{\star}\right) \equiv \mathcal{F}_{j}$.

Stressing that the problem objective only accounts the cost of the generation dispatch, at termination this iterative process returns the optimal solution of the full scenario-program $u_{N}^{\star}$. Moreover, the filtered subset $\mathcal{F}\left(u_{N}^{\star}\right)$ of cardinality $j+1$ is a support subset to the respective set of all $N$ scenarios. We should also clarify here that the feasibility assessment subproblems involved in step 1 are scenario-specific variants of the full problem statement, considering $1^{\text {st }}$-stage decisions as fixed and modified to search for the minimal degree of constraint violation (i.e., returning a zero optimal value for feasible combinations of $1^{\text {st }}$-stage decisions and injection scenario). We refer the reader to appendix $\mathrm{C}$ for the detailed statement of this feasibility assessment problem.

\section{Backward-pass: eliminating dominated scenarios}

As a second step, we search the filtered set $\mathcal{F}\left(u_{N}^{\star}\right)$ for dominated scenarios, that can be removed without any impact on the optimal $1^{\text {st }}$-stage decisions. Notice that such an eventuality may occur due to the iterative progression of the forwardpass. Indeed, the feasibility domain of a scenario filtered at a latter step $n>0$ may encompass the feasibility domain(s) of other scenario(s) already in the subset, filtered at steps $m<n$. To check for dominated scenarios we adopt the proposal from $\S$ II of [10], and, going through the filtering sequence $n=0, \ldots, j-1$ :

1) solve a scenario-program vs subset $\mathcal{F}\left(u_{N}^{\star}\right) \backslash i_{n}$ (i.e., excluding the concerned scenario from the filtered subset),

2) if the resulting $1^{\text {st }}$-stage decisions match those of the already known optimal solution $u_{n}^{\star}=u_{N}^{\star}$, update the filtered subset as $\mathcal{F}\left(u_{N}^{\star}\right)=\mathcal{F}\left(u_{N}^{\star}\right) \backslash i_{n}$,

3) update the scenario-program support cardinality as $k=$ $\left|\mathcal{F}\left(u_{N}^{\star}\right)\right|$.

\section{CASE STUdy RESUlts}

\section{A. Test-system description}

We adopt the test-system presented in [11], which is a modified version of the IEEE 30 bus system including 7 bus breakers (at nodes 4, 6, 7, 12, 21, 24, 30) as well as 3 phase-shifting transformers (at branches 22, 28, 33) and we consider $\mathrm{N}-1$ security w.r.t. the loss of transmission elements only and while considering a single forthcoming period only. The contingency list under consideration includes 33 single transmission element outages, excluding 8 elements whose outage (given the presence of PSTs) would bring the system to an islanded condition. To generate credible injection scenarios we employ, as in [13], a load forecast error model consisting of a zero mean, normally distributed global error term (i.e., common to all system loads) and a zero mean, normally distributed nodal error term. That is, under scenario $i$ the load demand at any network node $n$ is assumed as $d_{n}^{i}=\left(1+\epsilon_{0}^{i}+\epsilon_{n}^{i}\right) \cdot \hat{d}_{n}^{i}$, where, $\hat{d}_{n}^{i}$ represents the respective forecast value, $\epsilon_{0}^{i}$ is a scalar modeling the global error and $\epsilon_{n}^{i}$ is a scalar modeling the nodal error. The standard deviation of the global error term is assumed to be equal to 0.015 while the standard deviation of the nodal error term is, for any network node, assumed equal to 0.05 .

In the context of this problem, the upper bound on constraint violation probability as per (1) should be interpreted as an upper bound on the probability of failing to operate the system with $\mathrm{N}-1$ security, given the uncertainty on the nodal load demand. In what follows, we evaluate such upper bound while setting the confidence parameter as $\beta=0.0001$. Moreover, given optimal $1^{\text {st }}$-stage decisions, we also perform Monte Carlo assessments to empirically evaluate the probability of failing to meet the $\mathrm{N}-1$ criterion in operation, using a set of 10000 injection scenarios.

\section{B. Reference results}

To facilitate the presentation of our findings, let us start by introducing the solution to the problem in question, while considering a set of 50 nodal demand scenarios.

First, table I reports on an indicative single problem instance, used here to also explain the functionalities of our developed algorithm. The $1^{\text {st }}$-row of such table shows the problem optimal objective value, while the cardinalities of the support sets identified after the forward-and backward-pass of 
TABLE I

REFERENCE RESULT $v s 50$ SCENARIOS

\begin{tabular}{c|c}
\hline Cost $(\$)$ & 592.245 \\
\hline Filtered support cardinality & 6 \\
Reduced support cardinality & 4 \\
\hline$\varepsilon-$ upper bound & 0.4252 \\
$\varepsilon-$ empirical & 0.0526 \\
\hline
\end{tabular}

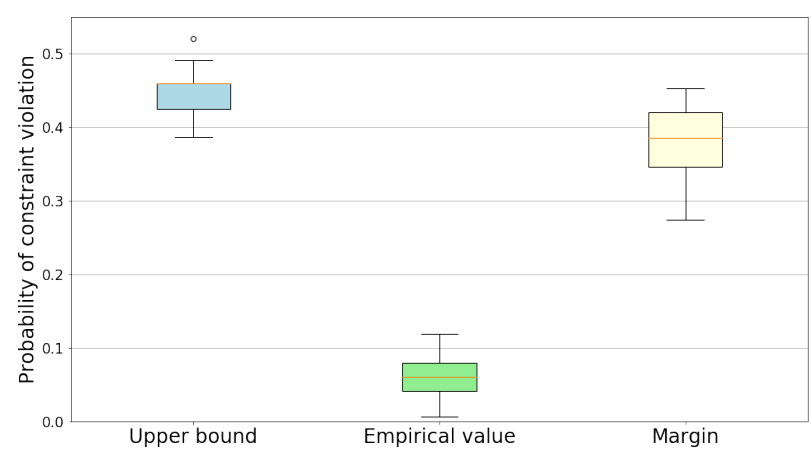

Fig. 2. 50 scenario-program constraint violation probability

the iterative algorithm are listed in the table $2^{\text {nd }}$ and $3^{\text {rd }}$ rows. Our detailed results indicate that the final support set retained after the backward-pass includes the lowest net load realization and three scenarios with active constraints on post-contingency power flow and on the coupling of pre- and post-contingency changes on breaker positions. The two dominated scenarios not retained after the forward pass are the forecast (i.e., no error) realization used to initiate the iterative procedure as well as the scenario with the greatest net load realization.

Most notably the two last rows of Table 1 present respectively the theoretical upper bound on constraint violation probability as per (1) as well as the empirical value of the probability of not being N-1 secure as per the ex-post Monte Carlo simulations. Examining such results, one could well argue that for the problem in question, a set of 50 scenarios appears too small to practically exploit the theoretical result from [10]. Indeed, holding a theoretical guarantee that the chance of complying with the $\mathrm{N}-1$ criterion is at most $\sim 60 \%$, appears to have limited practical relevance as power system security standards would typically be more restrictive. Be that as it may, what is more important to underline here is the vast difference between the theoretical upper bound and the empirically evaluated probability value (last row). Indeed, the latter value is one order of magnitude smaller than the former.

To further investigate the tightness of the theoretical upper bound, we created a total of 20 problem instances while considering sets of 50 nodal demand scenarios. The boxplots presented in Figure 2 summarize the empirical probability of failing to meet the $\mathrm{N}-1$ criterion and its respective upper bound. Such figures indeed confirm that the theoretical upper bound is in practice not tight for the problem in question. On average the ratio of the empirical violation probability to its

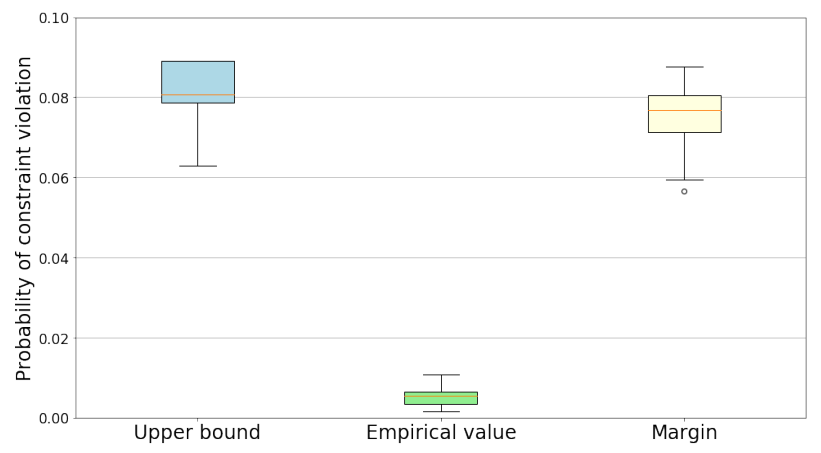

Fig. 3. 500 scenario-program constraint violation probability

theoretical upper bound is as small as $14.03 \%$. The difference between these two quantities per instance is summarized as 'Margin' in Figure 2, It is important to notice here that two factors are critical in computing a tight bound, namely both (a) the size $k$ of the support subset and (b) the size $N$ of the full scenario set of the stochastic program. Concerning the former, we must underline that a computationally exhaustive bruteforce approach, providing an explicit guarantee of returning a minimal and irreducible support set, is unattainable in practice. With this in mind, we continue the analysis by focusing on the second factor, namely the size of the problem scenario set.

\section{On the size of the problem scenario set}

In order to study the relationship between the size of the scenario-program and its respective constraint violation probability, we have resolved the 20 problem instances from subsection IV-B after a tenfold increase of the size of the considered set of scenarios. Precisely, per problem instance, we add 450 different scenarios along with the original 50 , to increase the scenario set size to 500. Figure 3 presents the updated boxplots on the theoretical upper bound and empirical probability of violating the $\mathrm{N}-1$ criterion in operation, as well as on the difference between these two quantities which is labeled as 'Margin'.

The decrease in the order of magnitude for both quantities is evident and consistent with the intuitive understanding that the $1^{\text {st }}$-stage decisions from the larger set scenario-program should indeed generalize better. Concerning the theoretical upper bound, the decrease implies that the cardinality of the support set identified by our iterative algorithm grows weakly with the size of the problem scenario set. Such feature is of course problem specific and relates to the fact that given reasonable power injection variability, few scenarios should be expected to be binding the power flow constraints (while dominating all others). On average, the case-by-case increase of the support set size was found to be equal to 0.4. Moreover, with respect to the values from the 50 scenario instances, the average reduction of the constraint violation probability upper bound was found equal to $81.70 \%$ while for the empirically evaluated probability the average reduction was notably computed at $86.38 \%$. 
TABLE II

Average Computational Time BREAKdown (SEC)

\begin{tabular}{c||c|cc}
\hline Problem Size & Total & Forward-pass & Backward-pass \\
\hline 50 & 1431 & 683.7 & 747.3 \\
500 & 2288.8 & 1578 & 710.8 \\
\hline
\end{tabular}

To pursue the present analysis, we turn again our attention on the tightness of the constraint violation probability upper bound. The bound is anticipated to be less tight since, as mentioned, on average its value is reduced less with respect to the empirical probability value. Indeed, the average ratio of the empirical violation probability to its theoretical upper bound is reduced to $6.75 \%$. The implication seems to be that a considerably larger scenario set would be needed for computing a lower constraint violation probability upper bound from (1). Just to give an order of magnitude, in average the support set of the considered scenario-program with 500 scenarios has 5 members and the respective average empirical probability of constraint violation was found equal to 0.00546 . Using such values in (1), the indicative problem size would be 11250 scenarios.

Given that the theoretical constraint violation probability bound was shown to be generally loose, the practical interest in solving such a large scale scenario-program depends on the computational burden. To inform such considerations, table II presents the breakdown of the average computational time to solve the 50 and 500 scenario problem instances on a personal computer featuring a 4-core, $4.2 \mathrm{GHz}$ processor and $24 \mathrm{~GB}$ of RAM. With the increase in problem size, the main increase is in the computational time required for the forward-pass of the algorithm. A main part of this step assesses (in parallel) all problem scenarios to identify constraint violations, hence it logically takes more time for larger problem instances. The computational time spent for the backward-pass of the algorithm is comparable between the two problem instances. This is attributable to the fact that the size of the scenario sets identified by the forward-pass is also comparable between the two problem instances. We also note that the backwardpass (employed only to obtain a more tight bound on the constraint violation probability) is computationally inexpensive in relation to the empirical evaluation with Monte Carlo simulations. In our experiments, the average computational time for a Monte Carlo simulation using 10000 realizations was 6947.2 seconds.

Finally, for completeness, we should mention that the difference in economic cost between the 500 scenario and the 50 scenario problem instances is negligible. On average, over the 20 trials the solution of the former is more costly by $0.11 \%$.

\section{On discrete grid flexibility}

To finish our analysis, we emphasize on the power system perspective and particularly the grid flexibility offered by the bus-splitting breakers. To do so, we solve a modified version of the initial scenario-program, enforcing a priori that all
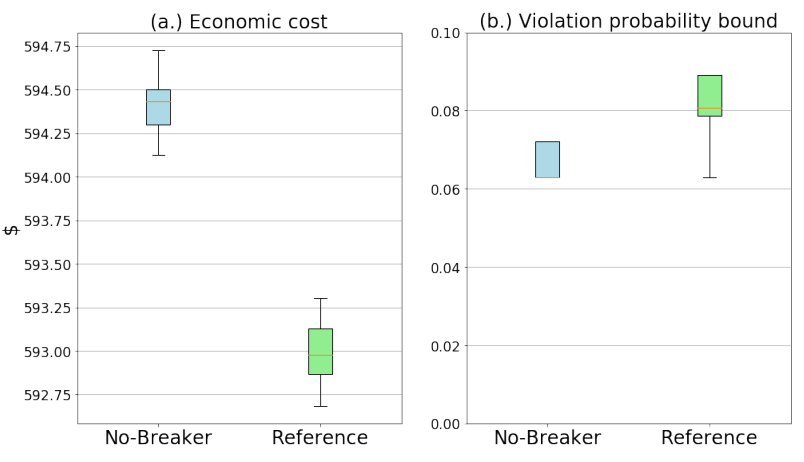

Fig. 4. 'No-breaker' vs 'reference' solution overview

bus splitting breakers should always remain closed. In what follows, we label such situation as 'no-breaker' setting and the original scenario-program as 'reference' setting. For the sake of comparability, we re-use the same 20 problem instances of 500 scenarios from subsection IV-C

First, we should briefly report on economic cost which, as anticipated, is increased while disregarding the grid flexibility. In particular, the average cost values of the 'no-breaker' and 'reference' settings are \$594.4 and \$593.0. Figure 4 a shows the respective cost value distributions while Figure $4 \mathrm{~b}$. has the value distributions for the upper bound on constraint violation probability. As seen in the latter figure, it appears that the constraint violation probability upper bound is lower for the 'no-breaker' setting with respect to the 'reference'. On a case-by-case basis, the average difference was found equal to 0.0145 . For scenario-programs considering the same sets of uncertainty realizations, the implication is that the support set to the optimal solution of the 'no-breaker' setting is systematically smaller than the support set to the optimal solution of the 'reference' setting. Indeed, on average the latter was found to include 1.65 additional members than the former. In our detailed results, we have identified additional scenarios that need to be kept in the support set of the 'reference' setting in order to optimally set the $1^{\text {st }}$-stage (i.e., intact network state) breaker position decision variables. These variables are of course fixed by default in the 'no-breaker' setting.

Comparing the empirical probability of violating the $\mathrm{N}-1$ criterion, Figure 5, we however found that the solutions to the 'reference' setting turn out to be in practice slightly more reliable, with an average reduction in the empirical constraint violation probability of 0.0025 (or, on average $25.05 \%$ of the 'no-breaker' empirical constraint violation probability). In other words, our finding here indicates that the constraint violation probability upper bound is less tight in the 'reference' setting. This may be explained by two factors. First, as discussed, the 'reference' setting has a higher upper bound on the constraint violation probability owing to the larger subset of support scenarios. At the same time, owing to the additional flexibility of adjusting the network topology once the power injection/demand scenario has been realized, it has a lower empirical constraint violation probability.

In any case, from a power systems standpoint, the results 


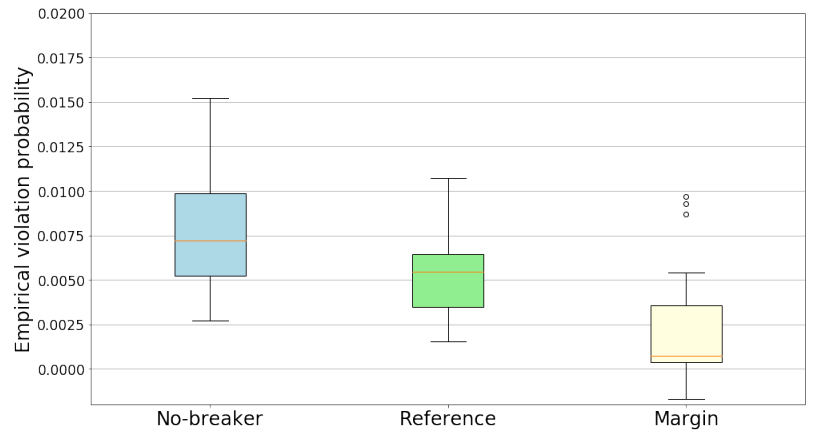

Fig. 5. 'No-breaker' $v s$ 'reference' empirical constraint violation probability

indeed showcase that grid flexibility contributes to greater economic efficiency as well as enhanced operational reliability. Thus, these results are in support of our interest towards leveraging discrete grid flexibility in chance-constrained operational planning.

\section{CONCLUding REMARKS}

In this work we studied the generalization property of scenario-based power system operation planning decisions, that is the probability of achieving N-1 secure operation with respect to unseen realizations from the probability distribution of uncertain power injections that is also used to generate the set of scenarios used to compute an optimal solution. We focused on an planning problem explicitly modeling grid flexibility, with the interest in progressively developing a practical chance-constrained decision making framework capable of exploiting the respective discrete power flow controls. Our analysis indicates the potential utility of grid flexibility, enabling to reduce the economic cost of operation decisions while increasing the probability of achieving N-1 secure operation.

Building on a recent result from the general scenario theory, we analyzed the theoretical upper bound of the probability in question along with its respective empirically estimated value. Concerning the theoretical upper bound, we have first observed that the size $k$ of the support-sets that we could construct with our greedy growing-pruning approach grows quite weakly with the size of the scenario-set used while searching to identify optimal operation planning decisions. In other words, increasing the number of scenarios taken into account leads to a stronger guarantee on the probability of achieving $\mathrm{N}-1$ security. In the more general context of power system operational planning, and given a reasonable range of injection variability, this condition should be anticipated to remain valid, as evidenced from the vast literature exploiting the concept of umbrella (a.k.a. dominant) injection realizations and contingencies in algorithmic approaches to security constrained optimal power flow. We have however also observed that the theoretical upper bound in question appears to be generally quite conservative, and potentially even more conservative in the presence of grid flexibility. Such issue is not to be underestimated, as relying on the theoretical probability bound could in practice imply taking unnecessary costly operation planning decisions.

To conclude, we underline that both the theoretical upper bound as well as the empirically estimated probability values were found to systematically decrease while growing the size of the scenario-set. These results imply the potential to solve chance-constrained operation planning problems while taking into account grid flexibility in the style of sequential randomization [14]. Indeed, in principle, one may algorithmically achieve a set target on the constraint violation probability by progressively increasing the size of the scenario set under consideration. We will explore such potential in the subsequent stage of this research effort. The first practical challenge to further study is how to optimally trade-off the easier to evaluate, yet conservative, theoretical upper bound and the more accurate, yet computationally more costly, empirical estimation.

\section{ACKNOWLEDGEMENTS}

The authors would like to thank H. Djelassi for providing detailed case study parameters. The authors thank the Isaac Newton Institute for Mathematical Sciences, University of Cambridge, for support and hospitality during the programme Mathematics of Energy Systems where work on this paper was initiated. This work was supported by EPSRC grant no EP/R014604/1. The authors also thank C. Vournas for hospitality at the National Technical University of Athens. LW acknowledges the support of F.R.S.-FNRS for his sabbatical year and the Simons Foundation for his fellowship during his stay at the Isaac Newton Institute.

\section{REFERENCES}

[1] F. Capitanescu, "Critical review of recent advances and further developments needed in AC optimal power flow," Electric Power Systems Research, vol. 136, pp. $57-68,2016$.

[2] M. Vrakopoulou, K. Margellos, J. Lygeros, and G. Andersson, "A probabilistic framework for reserve scheduling and N-1 security assessment of systems with high wind power penetration," IEEE Transactions on Power Systems, vol. 28, no. 4, pp. 3885-3896, Nov 2013.

[3] M. Vrakopoulou, M. Katsampani, K. Margellos, J. Lygeros, and G. Andersson, "Probabilistic security-constrained AC optimal power flow," in 2013 IEEE Grenoble Conference, June 2013, pp. 1-6.

[4] M. C. Campi, S. Garatti, and M. Prandini, "The scenario approach for systems and control design," Annual Reviews in Control, vol. 33, no. 2, pp. $149-157,2009$.

[5] L. Roald, F. Oldewurtel, T. Krause, and G. Andersson, "Analytical reformulation of security constrained optimal power flow with probabilistic constraints," in 2013 IEEE Grenoble Conference, June 2013, pp. 1-6.

[6] L. Roald and G. Andersson, "Chance-constrained AC optimal power flow: Reformulations and efficient algorithms," IEEE Transactions on Power Systems, vol. 33, no. 3, pp. 2906-2918, May 2018.

[7] K. Sundar, H. Nagarajan, L. Roald, S. Misra, R. Bent, and D. Bienstock, "Chance-constrained unit commitment with n-1 security and wind uncertainty," IEEE Transactions on Control of Network Systems, pp. 1-1, 2019.

[8] J. F. Marley, M. Vrakopoulou, and I. A. Hiskens, "An AC-QP optimal power flow algorithm considering wind forecast uncertainty," in 2016 IEEE Innovative Smart Grid Technologies - Asia (ISGT-Asia), Nov 2016, pp. 317-323.

[9] M. C. Campi, S. Garatti, and F. A. Ramponi, "Non-convex scenario optimization with application to system identification," in 2015 54th IEEE Conference on Decision and Control (CDC), Dec 2015, pp. 40234028. 
[10] - "A general scenario theory for nonconvex optimization and decision making," IEEE Transactions on Automatic Control, vol. 63, no. 12, pp. 4067-4078, Dec 2018.

[11] H. Djelassi, S. Fliscounakis, A. Mitsos, and P. Panciatici, "Hierarchical programming for worst-case analysis of power grids," in 2018 Power Systems Computation Conference (PSCC), June 2018, pp. 1-7.

[12] B. Zeng and L. Zhao, "Solving two-stage robust optimization problems using a column-and-constraint generation method," Operations Research Letters, vol. 41, no. 5, pp. 457 - 461, 2013

[13] L. Duchesne, E. Karangelos, and L. Wehenkel, "Using machine learning to enable probabilistic reliability assessment in operation planning," in 2018 Power Systems Computation Conference (PSCC), June 2018, pp. $1-8$.

[14] M. Chamanbaz, F. Dabbene, R. Tempo, V. Venkataramanan, and Q. Wang, "Sequential randomized algorithms for convex optimization in the presence of uncertainty," IEEE Transactions on Automatic Control, vol. 61, no. 9, pp. 2565-2571, Sep. 2016.

\section{APPENDIX}

\section{A. Nomenclature}

The main mathematical symbols used in this appendix are defined as follows. Others may be defined as needed within the text.

Indices \& sets:

$b \in \mathcal{B} \quad$ bus breakers;

$c \in \mathcal{C} \quad$ contingencies (including the pseudo-contingency of no outage, denoted by the index $c=0$ ) ;

$g \in \mathcal{G} \quad$ dispatchable generating units

$i \in \mathcal{I} \quad$ injection scenarios;

$\ell \in \mathcal{L} \quad$ transmission lines;

$n \in \mathcal{N}$ nodes;

$s \in \mathcal{S} \quad$ phase shifting transformers;

$e \in \mathcal{E} \quad$ generic transmission element (i.e., transmission line $\ell$, phase shifting transformer $s$ or bus breaker $b$ ) with superset $\mathcal{E} \equiv \mathcal{B} \cup \mathcal{L} \cup \mathcal{S}$.

$N b$ : Subscripts will be used to define subsets wherever needed (e.g., subset $\mathcal{G}_{n} \subseteq \mathcal{G}$ shall denote the generating units connected at node $n \in \mathcal{N})$.

\section{Continuous Variables:}

$a_{g} \quad$ AGC participation factor of generating unit $g$;

$p_{g} \quad$ dispatch of generating unit $g$;

$f_{e}^{i, c} \quad$ power flow through transmission element $e \in\{b, \ell, s\}$ under injection scenario $i$ and contingency $c$;

$\theta_{n}^{i, c} \quad$ voltage angle at node $n$ under injection scenario $i$ and contingency $c$;

$d \theta_{s}^{i, c} \quad$ shift of phase shifting transformer $s$ under injection scenario $i$ and contingency $c$;

$q_{e}^{i, c} \quad$ fictitious flow through transmission element $e \in\{b, \ell, s\}$ under injection scenario $i$ and contingency $c$ (full connectivity variable).

Binary Variables:

$z_{b}^{i, c} \quad$ status of bus breaker $b$ under injection scenario $i$ and contingency $c$;

$d z_{b}^{i, c} \quad$ indicator showing a change in the status of bus breaker $b$ under injection scenario $i$ and contingency $c \in \mathcal{C} \backslash 0$ with respect to the no outage pseudo-contingency $(c=0)$;

$\chi_{s, j}^{i, c} \quad$ indicator of phase shifting transformer $s$ operating mode under injection scenario $i$ and contingency $c$, for $j \in$ $[1 ; 2 ; 4 ; 5]$.

\section{Parameters.}

$c_{g} \quad$ marginal running cost of generating unit $g$;

$\underline{p}_{g} \quad$ minimum stable generation of generating unit $g$;

$\overline{\bar{p}}_{g}^{g} \quad$ capacity of generating unit $g$;

$\hat{d}_{n} \quad$ forecasted active power demand at node $n$;

$d_{n}^{i} \quad$ active power demand at node $n$ under injection scenario $i$;

$\bar{f}_{e} \quad$ rated capacity of transmission element $e \in\{b, \ell, s\}$;

$X_{e} \quad$ reactance of transmission element $e \in\{b, \ell, s\}$; $\beta_{n, e} \quad$ element of the flow incidence matrix, taking a value of one if node $n$ is the sending node of transmission element $e \in$ $\{b, \ell, s\}$, a value of minus one if node $n$ is the receiving node transmission element $e$ or phase shifting transformer $s$, and a zero value otherwise;

$y_{s} \quad$ activation threshold of phase shifting transformer $s$;

$\overline{d \theta}_{s} \quad$ maximum shift of phase shifting transformer $s$;

$d \theta_{s} \quad$ minimum shift of phase shifting transformer $s$;

$\frac{d u_{e}^{c}}{e} \quad$ availability of transmission element $e$ under contingency $c$;

$\zeta \quad$ maximum admissible number of post-contingency topological changes;

$M \quad$ a large constant.

\section{B. Scenario-program mathematical statement}

The mathematical statement of the scenario-program under consideration is as shown in 2,32 .

$\min \sum_{g \in \mathcal{G}} c_{g} \cdot p_{g}$

for all generating units, $g \in \mathcal{G}$ :

$$
\begin{aligned}
& 0 \leq a_{g} \leq 1, \\
& \underline{p}_{g} \leq p_{g} \leq \bar{p}_{g}, \\
& \underline{p}_{g} \leq p_{g}+a_{g} \cdot \sum_{n \in \mathcal{N}}\left(d_{n}^{i}-\hat{d}_{n}\right) \leq \bar{p}_{g}, \quad \forall i \in \mathcal{I} ;
\end{aligned}
$$

for all nodes, injections \& contingencies $(n, i, c) \in \mathcal{N} \times \mathcal{I} \times \mathcal{C}$ :

$$
\sum_{g \in \mathcal{G}_{n}}\left[p_{g}+a_{g} \cdot \sum_{n \in \mathcal{N}}\left(d_{n}^{i}-\hat{d}_{n}\right)\right]=d_{n}^{i}+\sum_{e \in \mathcal{E}} \beta_{n, e} \cdot f_{e}^{i, c} ;
$$

for all lines, injections \& contingencies $(\ell, i, c) \in \mathcal{L} \times \mathcal{I} \times \mathcal{C}$ :

$$
\begin{aligned}
& f_{\ell}^{i, c}-\frac{u_{\ell}^{c}}{X_{\ell}} \sum_{n \in \mathcal{N}} \beta_{n, \ell} \cdot \theta_{n}^{i, c}=0, \\
& -\bar{f}_{\ell} \leq f_{\ell}^{i, c} \leq \bar{f}_{\ell}, \\
& -u_{\ell}^{c} \cdot M \leq q_{\ell}^{i, c} \leq u_{\ell}^{c} \cdot M
\end{aligned}
$$

for all phase-shifting transformers, injections and contingencies, $(s, i, c) \in \mathcal{S} \times \mathcal{I} \times \mathcal{C}:$

$$
\begin{aligned}
& f_{s}^{i, c}-\frac{u_{\ell}^{c}}{X_{s}} \sum_{n \in \mathcal{N}} \beta_{n, s} \cdot\left(\theta_{n}^{i, c}+d \theta_{s}^{i, c}\right)=0, \\
& \left(\chi_{s, 1}^{i, c}-1\right) \cdot M+\overline{d \theta}_{s} \leq d \theta_{s}^{i, c} \leq \overline{d \theta}_{s}+\left(1-\chi_{s, 1}^{i, c}\right) \cdot M, \\
& -\frac{u_{s}^{c}}{X_{s}} \cdot \sum_{n \in \mathcal{N}} \beta_{n, s} \cdot \theta_{n}^{i, c}-y_{s} \leq \chi_{s, 1}^{i, c} \cdot M, \\
& \frac{u_{s}^{c}}{X_{s}} \cdot \sum_{n \in \mathcal{N}} \beta_{n, s} \cdot \theta_{n}^{i, c}+y_{s} \leq\left(1-\chi_{s, 1}^{i, c}\right) \cdot M, \\
& \left(\chi_{s, 2}^{i, c}-1\right) \cdot M \leq d \theta_{s}^{i, c} \leq \overline{d \theta}_{s}+\left(1-\chi_{s, 2}^{i, c}\right) \cdot M, \\
& -\frac{u_{s}^{c}}{X_{s}} \cdot \sum_{n \in \mathcal{N}} \beta_{n, s} \cdot \theta_{n}^{i, c}-y_{s} \leq\left(1-\chi_{s, 2}^{i, c}\right) \cdot M, \\
& \frac{u_{s}^{c}}{X_{s}} \cdot \sum_{n \in \mathcal{N}} \beta_{n, s} \cdot \theta_{n}^{i, c}+y_{s} \leq\left(1-\chi_{s, 2}^{i, c}\right) \cdot M, \\
& -\sum_{j \in\{1 ; 2 ; 4 ; 5\}} \chi_{s, j}^{i, c} \cdot M \leq d \theta_{s}^{i, c} \leq \sum_{j \in\{1 ; 2 ; 4 ; 5\}} \chi_{s, j}^{i, c} \cdot M, \\
& \left(\chi_{s, 4}^{i, c}-1\right) \cdot M+\underline{d \theta}_{s} \leq d \theta_{s}^{i, c} \leq\left(1-\chi_{s, 4}^{i, c}\right) \cdot M, \\
& -\frac{u_{s}^{c}}{X_{s}} \cdot \sum_{n \in \mathcal{N}} \beta_{n, s} \cdot \theta_{n}^{i, c}+y_{s} \leq\left(1-\chi_{s, 4}^{i, c}\right) \cdot M, \\
& \frac{u_{s}^{c}}{X_{s}} \cdot \sum_{n \in \mathcal{N}} \beta_{n, s} \cdot \theta_{n}^{i, c}-y_{s} \leq\left(1-\chi_{s, 4}^{i, c}\right) \cdot M, \\
& \left(\chi_{s, 5}^{i, c}-1\right) \cdot M+\underline{d \theta}_{s} \leq d \theta_{s}^{i, c} \leq \underline{d \theta_{s}+\left(1-\chi_{s, 5}^{i, c}\right) \cdot M ;} ;
\end{aligned}
$$




$$
\begin{aligned}
& \frac{u_{s}^{c}}{X_{s}} \cdot \sum_{n \in \mathcal{N}} \beta_{n, s} \cdot \theta_{n}^{i, c}-y_{s} \leq \chi_{s, 5}^{i, c} \cdot M, \\
& \frac{u_{s}^{c}}{X_{s}} \cdot \sum_{n \in \mathcal{N}} \beta_{n, s} \cdot \theta_{n}^{i, c}-y_{s} \geq\left(\chi_{s, 5}^{i, c}-1\right) \cdot M, \\
& q_{s}^{i, c}=0
\end{aligned}
$$

for all breakers, injections \& contingencies $(b, i, c) \in \mathcal{B} \times \mathcal{I} \times \mathcal{C}$ :

$$
\begin{aligned}
& -z_{b}^{i, c} \cdot M \leq f_{b}^{i, c} \leq z_{b}^{i, c} \cdot M \\
& -z_{b}^{i, c} \cdot M \leq q_{b}^{i, c} \leq z_{b}^{i, c} \cdot M \\
& -d z_{b}^{i, c} \leq z_{b}^{i, c}-z_{b}^{i, 0} \leq d z_{b}^{i, c}
\end{aligned}
$$$$
\left(1-z_{b}^{i, c}\right) \cdot M \leq \sum_{n \in \mathcal{N}} \beta_{n, b} \cdot \theta_{n}^{i, c} \leq\left(1-z_{b}^{i, c}\right) \cdot M,
$$

for all injections \& contingencies $(i, c) \in \mathcal{I} \times \mathcal{C}$ :

$$
\begin{aligned}
& \sum_{b \in \mathcal{B}} d z_{b}^{i, c} \leq \zeta, \\
& \theta_{n_{0}}^{i, c}=0, \\
& \sum_{e \in \mathcal{E}_{n_{0}}} q_{e}^{i, c}+1-|\mathcal{N}|=0, \\
& \sum_{e \in \mathcal{E}_{n}} q_{e}^{i, c}+1=0, \quad \forall n \in \mathcal{N} \backslash n_{0} .
\end{aligned}
$$

Objective function (2) seeks to minimize the cost of the generation dispatch. Inequalities (3) set the range of admissible values for the participation factors of generating units, while expressions 4 - 5 impose the minimum stable generation and maximum generation capacity limits for the dispatch state and all considered uncertainty realizations respectively. Expressions (6- 8) are standard nodal power balance and power flow expressions of the contingency constrained optimal power flow problem under the DC power flow approximation. Inequalities (9) impose that any line on outage does not contribute to the network connectivity.

Similarly, constraints (10 - ??) define the active power flow through PSTs and impose the respective limits. The value of the phase-shift variable $\left(d \theta_{s}^{i, c}\right)$ appearing in 10 is set through constraint block (11 - 23), according to the magnitude and direction of the respective nodal voltage angle difference, as per the following five PST operating modes:

M1: negative flow below the respective activation limit $\Rightarrow$ inject the maximum positive phase angle difference to increase flow (11-13).

M2: flow equals the negative activation limit $\Rightarrow$ inject any admissible non-negative phase angle difference to increase flow $12-16$.

M3: flow within both activation limits $\Rightarrow$ do nothing (17).

M4: flow equals the positive activation limit $\Rightarrow$ inject any admissible non-positive phase angle difference to reduce flow $18-20$.

M5: positive flow above the respective activation limit $\Rightarrow$ inject the minimum negative phase angle difference to reduce flow 21- 23.

Notice that, for the sake of determining network connectivity PSTs need to be considered as disconnected (24).

The group of inequalities 25 - 27 models the opening/closing of breakers to split/merge the respective network nodes. First, inequalities 25 equate the voltage angles on the two sides of a closed breaker only. Next, inequalities (26) allow unrestricted power flow through a closed breaker, conversely no power flow through an open breaker. Finally, inequalities 27) are used to model the contribution of a closed breaker to the connectivity of the electrical network. Expressions 28] are used to count the number of topological changes in post-contingency states, with respect to the no outage pseudo-contingency state $(c=0)$. Further, constraint 29 imposes the applicable limit for such changes.

Equality constraints 30 set the network reference node $\left(n_{0}\right)$. At last, equalities 31.32 establish the full connectivity of the grid in pre- and post- contingency stages, by balancing the flow of the respective fictitious quantity.

\section{Feasibility assessment sub-problem mathematical state-} ment

The injection-specific feasibility assessment sub-problem is a relaxation of the full scenario-program 2232 , given fixed $1^{\text {st }}$-stage decision variables, annotated henceforth with a tilde $(\tilde{*})$. To derive such injection-specific relaxation, $\forall i \in \mathcal{I}$,

- non-negative slack variables $\left(\mu_{g}^{i}, \nu_{g}^{i}\right)$ relax generation lower \& upper bounds in (5), taking positive values to indicate that the generation - load mismatch cannot be balanced, as in,

for all generating units, $g \in \mathcal{G}$ :

$$
\begin{aligned}
& \mu_{g}^{i} \geq 0 \\
& \nu_{g}^{i} \geq 0 \\
& \underline{p}_{g}-\mu_{g}^{i} \leq \tilde{p}_{g}+\tilde{a}_{g} \cdot \sum_{n \in \mathcal{N}}\left(d_{n}^{i}-\hat{d}_{n}\right) \leq \bar{p}_{g}+\nu_{g}^{i} ;
\end{aligned}
$$

- non-negative slack variables $\left(\lambda_{\ell}^{i, c}\right)$ relax the transmission capacity ratings in (8), taking positive values to indicate that there is no feasible constrained power flow solution, as in,

for all lines \& contingencies $(\ell, c) \in \mathcal{L} \times \mathcal{C}$ :

$$
\begin{aligned}
& \lambda_{\ell}^{i, c} \geq 0, \\
& -\left(1+\lambda_{\ell}^{i, c}\right) \cdot \bar{f}_{\ell} \leq f_{\ell}^{i, c} \leq\left(1+\lambda_{\ell}^{i, c}\right) \cdot \bar{f}_{\ell} ;
\end{aligned}
$$

- similarly, non-negative slack variables $\left(\lambda_{s}^{i, c}\right)$ relax the PST ratings in (??), taking positive values to indicate that there is no feasible constrained power flow solution, as in,

for all phase-shifting transformers \& contingencies $(s, c) \in$ $\mathcal{S} \times \mathcal{C}:$

$$
\begin{aligned}
& \lambda_{s}^{i, c} \geq 0, \\
& -\left(1+\lambda_{s}^{i, c}\right) \cdot \bar{f}_{s} \leq f_{s}^{i, c} \leq\left(1+\lambda_{s}^{i, c}\right) \cdot \bar{f}_{s} ;
\end{aligned}
$$

- the problem objective seeks to minimize the sum of the aforementioned non-negative variables should take the zero value for feasible problem instances, as in,

$$
\min \sum_{g \in \mathcal{G}}\left(\mu_{g}^{i}+\nu_{g}^{i}\right)+\sum_{c \in \mathcal{C}}\left(\sum_{\ell \in \mathcal{L}} \lambda_{\ell}^{i, c}+\sum_{s \in \mathcal{S}} \lambda_{s}^{i, c}\right) .
$$

